\title{
Human CD39hi regulatory T cells present stronger stability and function under inflammatory conditions
}

\author{
Jian $\mathrm{Gu}^{1,4}$, Xuhao $\mathrm{Ni}^{1,4}$, Xiongxiong $\mathrm{Pan}^{1}$, Hao Lu${ }^{1}$, Yunjie Lu${ }^{1,2}$, Jie Zhao ${ }^{1}$, Song Guo Zheng ${ }^{3}$, \\ Keli L Hippen ${ }^{2}$, Xuehao Wang ${ }^{1}$ and Ling $\mathrm{Lu}^{1}$
}

Autoimmune diseases are characterized by an imbalance between regulatory $\mathrm{T}$ cells and effector T-cell subsets, such as Th1 and Th17 cells. Studies have confirmed that natural $\mathrm{CD} 4^{+} \mathrm{Foxp} 3^{+}$Tregs were unstable and dysfunctional in the presence of pro-inflammatory cytokines. In the current study, human CD39hi Tregs and CD39low Tregs were sorted from Tregs in vitro after 7 days of expansion. The functions of both Treg subsets were investigated under inflammatory conditions in vitro and in vivo. In the presence of IL-1 $\beta$ and IL-6, cultured CD4+CD39hi Tregs maintained stable forkhead box protein 3 expression, whereas CD4 ${ }^{+}$CD39low Tregs lost Foxp3 expression and trans-differentiated into Th1 or Th17 cells. Decreased IL-1ßR and IL-6R expression on the CD39hi Tregs was the primary mechanism responsible for Treg stability. In addition, reduced activation of downstream molecules, such as STAT1 and STAT3, through the modulation of $\mathrm{CpG}$ demethylation played an important role. Finally, human $\mathrm{CD} 4{ }^{+} \mathrm{CD} 39^{\text {hi }}$ Tregs but not $\mathrm{CD} 4^{+} \mathrm{CD} 39^{\text {low }}$ Tregs protected against xenograft versus host disease in model mice. These results strongly implied the physiological importance of CD39 expression and suggested that manipulation of CD39hi Tregs might represent a novel strategy for the treatment of autoimmune diseases.

Cellular \& Molecular Immunology (2017) 14, 521-528; doi:10.1038/cmi.2016.30; published online 4 July 2016

Keywords: CD39; stability; Tregs; xeno-GVHD

\section{INTRODUCTION}

Regulatory $\mathrm{T}$ cells (Tregs) play an essential role in maintaining immunologic homeostasis. ${ }^{1,2}$ Various autoimmune diseases in mice, including autoimmune diabetes, ${ }^{3}$ experimental arthritis ${ }^{4}$ and acute graft versus host disease (GvHD), ${ }^{5}$ can be treated with Tregs. Although their importance for proper immune regulation is undeniable, the stability of the Treg lineage has recently become a topic of debate. ${ }^{6}$ Large losses of the signature Treg transcription factor forkhead box protein 3 (Foxp3) and Treg functions have been observed under various inflammatory conditions.

CD39 is an ectoenzyme that hydrolyses adenosine triphosphate and adenosine diphosphate into adenosine monophosphate (AMP). ${ }^{7} \mathrm{CD} 39$ is primarily localized on the surfaces of endothelial cells and circulating platelets and has been implicated in the suppressive function of Tregs. ${ }^{8}$ However, the pathological importance of $\mathrm{CD} 39$ to the stability of the Foxp $3^{+}$population remains unclear.
Tregs can be expanded in a GMP facility by $>300$-fold, which is adequate for cell therapy $\left(1-10 \times 10^{6} / \mathrm{kg}\right)$. However, the methods used to improve the stability and functionality of this expanded population are still being devised. In this study, CD39hi and CD39low Tregs were isolated and cultured with the addition of interleukin (IL)- $1 \beta$ and IL-6. Xeno-GvHD models were used to examine the in vivo stability and functionality of the $\mathrm{CD} 39^{\mathrm{hi}}$ and CD39 ${ }^{\text {low }}$ Treg subsets. Our results strongly suggest that CD39hi Tregs may provide more effective clinical benefits for cell therapybased strategies to prevent and control autoimmune diseases.

\section{MATERIALS AND METHODS}

Treg isolation and expansion

Peripheral blood mononuclear cells (PBMCs) were prepared from the heparinized venous blood of healthy adult volunteers by Ficoll-Hypaque (GE, Fairfield, CT, USA) density gradient centrifugation. Human $\mathrm{CD} 4^{+} \mathrm{CD} 127^{-} \mathrm{T}$ cells were sorted by

\footnotetext{
${ }^{1}$ Translational Medicine Research Center, Affiliated Jiangning Hospital, and Liver Transplantation Center, First Affiliated Hospital, Nanjing Medical University, Nanjing 210029, China; ${ }^{2}$ University of Minnesota Cancer Center and Division of Bone Marrow Transplantation, Department of Pediatrics, University of Minnesota, Minneapolis, MN 55455, USA and ${ }^{3}$ Division of Rheumatology, Penn State Milton S. Hershey Medical Center, Hershey, PA 17033, USA ${ }^{4}$ These authors contributed equally to this work. Correspondence: Dr X Wang, MD or Dr L Lu, MD PhD, Translational Medicine Research Center, Affiliated Jiangning Hospital, and Liver Transplantation Center, First Affiliated Hospital, Nanjing Medical University, Nanjing 210029, China.

E-mail: wangxh@njmu.edu.cn or Ivling@njmu.edu.cn

Received: 9 January 2016; Revised: 5 May 2016; Accepted: 6 May 2016
} 
depleting CD8, CD19, CD14 and CD127 cells using an auto-MACS (Miltenyi, San Diego, CA, USA), whereas $\mathrm{CD} 4^{+} \mathrm{CD} 25^{+} \mathrm{CD} 127^{-}$ Tregs were sorted with anti-CD25 beads (Miltenyi, $>97 \%$ purity). The Tregs were activated and expanded using anti-CD3/CD28 beads (Dynal, 1 bead to 2 cells) and IL-2 (R\&D Systems, Los Angeles, CA, USA, $300 \mathrm{U} / \mathrm{ml}$ ) for 7 days. Then, the CD39hi and CD39low Foxp3 cells were sorted from the expanded Tregs.

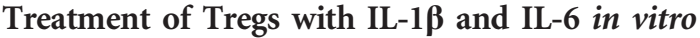

In some experiments, Tregs were re-stimulated with anti-CD3/28 beads (Dynal beads, 1 bead to 2 cells), IL-2 (R\&D Systems, 20 U/ $\mathrm{ml}) \pm \mathrm{IL}-6$ (R\&D Systems, $10-30 \mathrm{ng} / \mathrm{ml})$ and IL-1 $\beta$ (R\&D Systems, $10 \mathrm{ng} / \mathrm{ml}$ ) for 3 days. This low dose of IL-2 was chosen to facilitate the IL-1 $\beta$ and IL-6-mediated conversion of Tregs into Th17 cells. High doses of IL- 2 in culture have been shown to stabilize the expression of Foxp3 and inhibit the differentiation of Foxp $^{+}$Tregs. $^{9}$

\section{Suppressive assay in vitro}

PBMCs were prepared from the heparinized venous blood of healthy adult volunteers. The PBMCs were labelled with CFSE (Invitrogen, Carlsbad, CA, USA) and stimulated with anti-CD3 mAb-coated beads (Dynal) at a 1:1 bead to PBMC ratio. Tregs were added at varying ratios, and the cultures were incubated at $37^{\circ} \mathrm{C}$. On day 4 , the cells were stained with APC-conjugated antiCD8 and examined using flow cytometry.

\section{Intracellular staining by flow cytometry}

For intracellular cytokine staining, the cells were stimulated with phorbol myristate acetate $(0.25 \mu \mathrm{g} / \mathrm{ml})$ and ionomycin $(0.25 \mu \mathrm{g} / \mathrm{ml})$ for $5 \mathrm{~h}$ and brefeldin A $(5 \mu \mathrm{g} / \mathrm{ml})$ for $4 \mathrm{~h}$. Then, the cells were stained to assess surface markers, such as CD4 and CD25 (eBioscience, Santa Clara, CA, USA), followed by further fixation, permeabilization and staining to detect Foxp3, IL-17A, IFN- $\gamma$ and IL-6 (Biolegend, San Diego, CA, USA) expression.

\section{Quantitative real-time PCR}

RNA was extracted from the harvested cells using the RNA Simple Total RNA kit (Tiangen Biotech, Beijing, China) according to the manufacturer's instructions. cDNA was synthesized using the RTMaster Mix (TaKaRa, Mountain View, CA, USA) and amplified by quantitative reverse transcription PCR (RT-PCR) in an ABI Prism system (Applied Biosystems, Foster City, CA, USA). The data were analysed using the relative gene expression method and normalized to the glyceraldehyde-3-phosphate dehydrogenase $\mathrm{Ct}$ values in the samples. Each sample was measured in triplicate. The primer sequences used for the PCR amplification were as follows:

IL-17, 5' -TGAAAACACAGAAGTAACGTCCG-3' and 5'-CCCA GGAGGAAATTGTAATGGGA-3'; IL-1 $\beta$ receptor, 5' ${ }^{\prime}$ TACCACTC TGATTGTGGACTGC- $3^{\prime}$ and $5^{\prime}$-CCCCT;TCTCTGATTCGTTTG GAT-3'; and IL-6 receptor, 5'-CATTGCCATTGTTCTGAGGTTC-3' and 5'-AGTAGTCTGTATTGCGA-3'.

\footnotetext{
Xeno-GvHD model generation

NOD/scid/IL2r common $\gamma$ chain $^{-1-}$ (NOG) mice were obtained from Jackson Laboratory (Bar Harbor, ME, USA). The mice received a single dose of $200 \mathrm{cGy}$ of gamma irradiation from a
}

linear accelerator before the same-day injection of human PBMCs. Some irradiated mice received PBS instead of human PBMCs and served as the control group. All experiments were performed according to the guidelines of the Institutional Animal Committee of Nanjing Medical University.

\section{Western blotting analysis}

Proteins were extracted from the harvested cells and the protein concentration was determined using the BCA assay (Pierce, Boston, MA, USA). Protein samples $(30 \mu \mathrm{g})$ were resolved by SDS-polyacrylamide gel electrophoresis and transferred to a polyvinylidene difluoride membrane. The following antibodies were used: STAT1 (Cell Signalling Technology, Beverly, MA, USA, \#9170), STAT3 (Abcam, Cambridge, UK, Ab7966), p-STAT1 (Cell Signalling Technology, \#7649), and p-STAT3 (Abcam, Ab131103). The results were visualized with Kodak autoradiography film (Kodak XAR film, Molendinar, Japan).

\section{TSDR methylation status assay}

Genomic DNA was extracted using the DNA Extraction kit (Beyotime, Shanghai, China) and processed using the EZ DNA Methylation-Direct kit (Zymo Research, Irvine, CA, China) following the manufacturers' protocols. Purified bisulfite-treated DNA was used for the bisulfite-sequencing PCR with the following tregspecific demethylation region (TSDR) amplification PCR primers: 5'-TTG GGT TAA GTT TGT TGT AGG ATA G-3' and 5'-ATC TAA ACC CTA TTA TCA CAA CCC C-3'. The PCR products were purified and cloned; single clones were selected for sequencing. All sequencing results of the bisulfite-converted TSDR region were analysed on the BDPC DNA methylation analysis platform. The average methylation status of the $\mathrm{CpG}$ sites in the TSDR region were statistically analysed.

\section{Statistics}

Differences in the Kaplan-Meier survival curves were analysed with the log-rank test and other statistical comparisons with the indicated group. The statistical analyses were performed with Student's $t$-test or analysis of variance using the GraphPad Prism software (La Jolla, CA, USA).

\section{RESULTS}

Human CD39hi Tregs sustained higher Foxp3 expression levels and stronger suppressive abilities even in the presence of inflammatory cytokines in vitro

Tregs isolated from human PBMCs were cultured for 7 days with IL-2 and anti-CD3/CD28 beads. The cells were harvested and stratified into two populations as follows: cells with stronger CD39 expression (defined as CD39hi Tregs) and the remainder (defined as CD39low Tregs). After culture for 7 days, $87 \%$ of the cells were CD4 ${ }^{+}$ Foxp $3^{+}$; although most of the cells were CD39 positive, half of the cells expressed higher CD39 levels compared with the rest of the cells (Supplementary Figure 1A). The phenotype analysis indicated that Treg markers such as Foxp3 and Helios were similarly expressed in both subsets. CD39 ${ }^{\text {hi }}$ Tregs expressed higher PD-L1, CD25 and cytotoxic T lymphocyte-associated protein 4 (CTLA-4) levels but lower GITR and CD127 levels compared with the CD39low Tregs 
(Supplementary Figure 1B). The CD39hi Tregs secreted more IL-10 than the CD39low Tregs, although TGF- $\beta$ expression did not significantly differ between the groups (Supplementary Figure 1C). The CFSE co-culture assay was performed to estimate the suppressive ability of the two Treg subsets. Tregs were co-incubated with CFSE-labelled fresh PBMCs in the presence of anti-CD3 beads. The CD39hi Tregs showed stronger suppressive activity at any ratio compared with the CD39 ${ }^{\text {low }}$ Tregs (Figure 1a). As confirmed from previous reports, the combination of IL- $1 \beta$ and IL- 6 was important for trans-differentiation because either cytokine alone induced much less Th1/Th17 conversion. ${ }^{9}$ Without cytokine addition, the CD39hi and CD39 ${ }^{\text {low }}$ Tregs expressed similar levels of Foxp3; however, IL-1 $\beta$ and IL-6 stimulation downregulated the Foxp3 level in human CD39 ${ }^{\text {low }}$ Tregs (from $72.2 \pm 4.7$ to $36 \pm 3.6 \%$ ) compared with CD39hi Tregs (from $71.5 \pm 3.1$ to $62 \pm 4.2 \%$ ), which corresponded to decreased resistance to Th1 and Th17 cell conversion (Figure $1 b$ ). Because the suppressive function of Tregs is important for the potential therapeutic benefit, CFSE co-culture assays were performed to estimate the suppressive abilities of the CD39hi and CD39 low Tregs following the addition of inflammatory cytokines. DMSO or cytokine-treated Tregs were co-incubated with CFSE-labelled fresh PBMCs at a 1:5 ratio in the presence of anti-CD3 beads. Although no significant difference was detected in the suppressive functions of the CD39hi and CD39 $9^{\text {low }}$ Tregs under cytokine-free conditions, the CD39 ${ }^{\text {low }}$ Tregs lost their ability to suppress PBMC proliferation upon the addition of IL- $1 \beta$ and IL-6, whereas only a slight decrease in the CFSE dilution was observed in the CD39hi Treg group (Figure 1c).
Human CD39hi Tregs expressed less IL-1 $\beta$ R and IL-6R and became resistant to IL-1//IL-6-driven Th1 and Th17 conversion

IL-17A and IFN- $\gamma$ production in CD39low Tregs following IL-1 $\beta$ and IL-6 re-stimulation was elevated compared with the group restimulated without these cytokines. Interestingly, the trend in IL-17A and IFN- $\gamma$ expression observed in the CD39 ${ }^{\text {hi }}$ Tregs was similar under both the normal and inflammatory conditions (Figure 2a). The IL-1 $\beta$ and IL-6 receptors are necessary to bind the cytokines and activate downstream signals. Thus, we tested the expression of IL-1 $\beta R$ and IL-6R in the two Treg subsets under different conditions. Fresh Tregs expressed low levels of IL-6R, whereas IL-1 $\beta \mathrm{R}$ was not detected. The IL-1 $\beta \mathrm{R}$ level did not significantly change, but the IL-6R expression level increased upon TCR stimulation. Interestingly, IL-1 $\beta \mathrm{R}$ and IL-6R expression significantly increased following cytokine stimulation for 3 days in the CD39low Tregs, indicating that these cytokines regulated the CD39 $9^{\text {low }}$ Tregs through the expression and activation of their receptors. The expression levels of both receptors were only slightly increased on the activated and expanded CD39hi Tregs (Figure 2b). These results confirmed the previous findings in mouse Tregs and implied that the stability of Tregs was partially ascribed to the downregulation of IL- $1 \beta \mathrm{R}$ and IL-6R. ${ }^{10}$

\section{Stronger STAT1 and STAT3 phosphorylation and CpG} methylation of the Foxp3 gene was detected in CD39low Tregs compared with CD39hi ${ }^{\text {Tregs }}$

A previous study indicated that the functions of IL-1 $\beta$ and IL-6 depended on the activation of STAT1 and STAT3. ${ }^{11}$ To determine

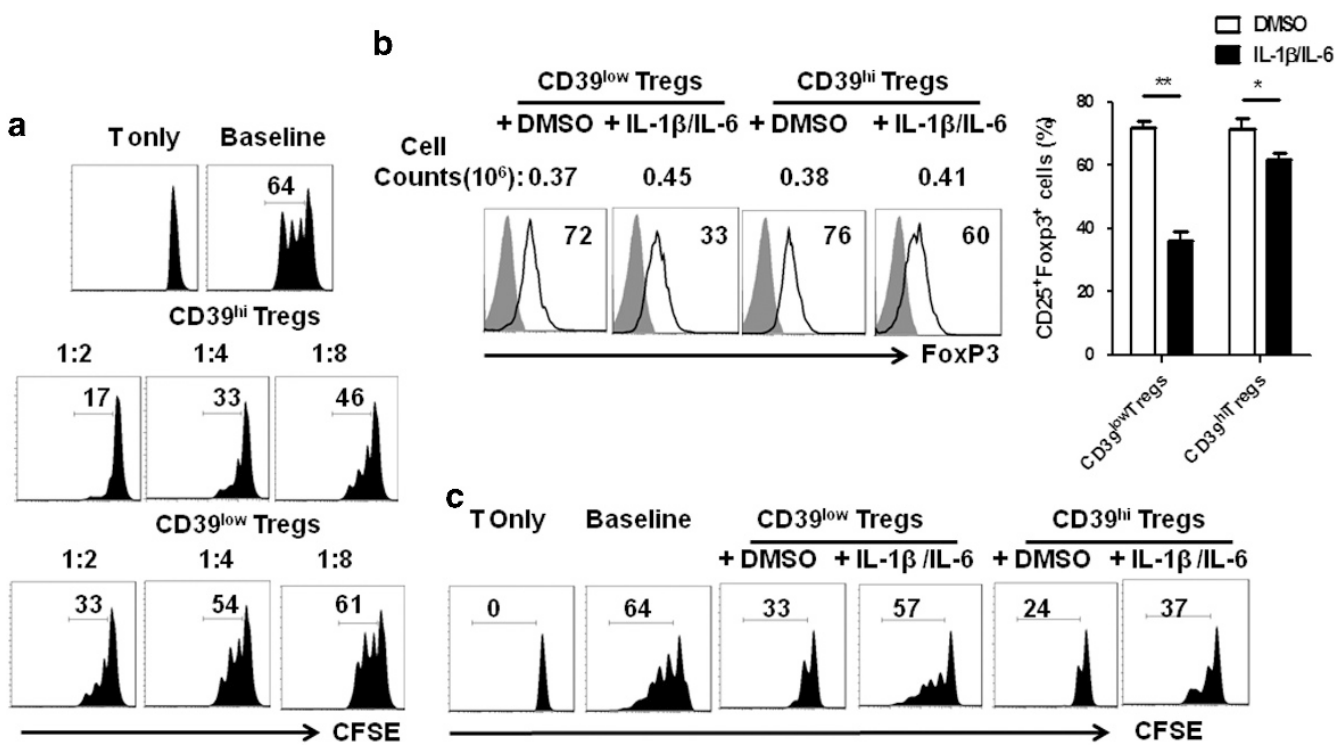

Figure 1 Human CD39hi Tregs sustained the Foxp3 expression level and suppressive ability even in the presence of inflammatory cytokines in vitro. CFSE-labelled PBMCs were co-cultured with the CD39hi and CD39low Treg subsets in the presence of anti-CD3 mAb-coated beads for 3 days. The cells were stained with an anti-human CD8 antibody and assessed with flow cytometry. (a) The suppressive activity of various primed cell subsets on CFSE-labelled CD8 ${ }^{+}$cells at different $\mathrm{T}$ suppressor to T-effector ratios is shown. $\mathrm{T}$ only refers to $\mathrm{T}$ cells without any other cell subsets or beads and the baseline indicates the co-culture of $T$ cells with beads but not Tregs. (b) Left panel: representative histogram of Foxp3 frequencies for both Treg subsets with or without the addition of inflammatory cytokines. Right panel: the percentage of $\mathrm{CD}_{2} 5^{+} \mathrm{Foxp}^{+} \mathrm{T}$ cells with or without the addition of inflammatory cytokines. The data are the mean $\pm \mathrm{s}$.e.m. of four independent experiments. (c) Representative histogram plot of the suppressive ability of the Treg subsets for CFSE-labelled CD8 ${ }^{+}$cells examined by flow cytometry. The result is representative of four independent experiments. ${ }^{*} P<0.05 ;{ }^{*} P<0.01$. 

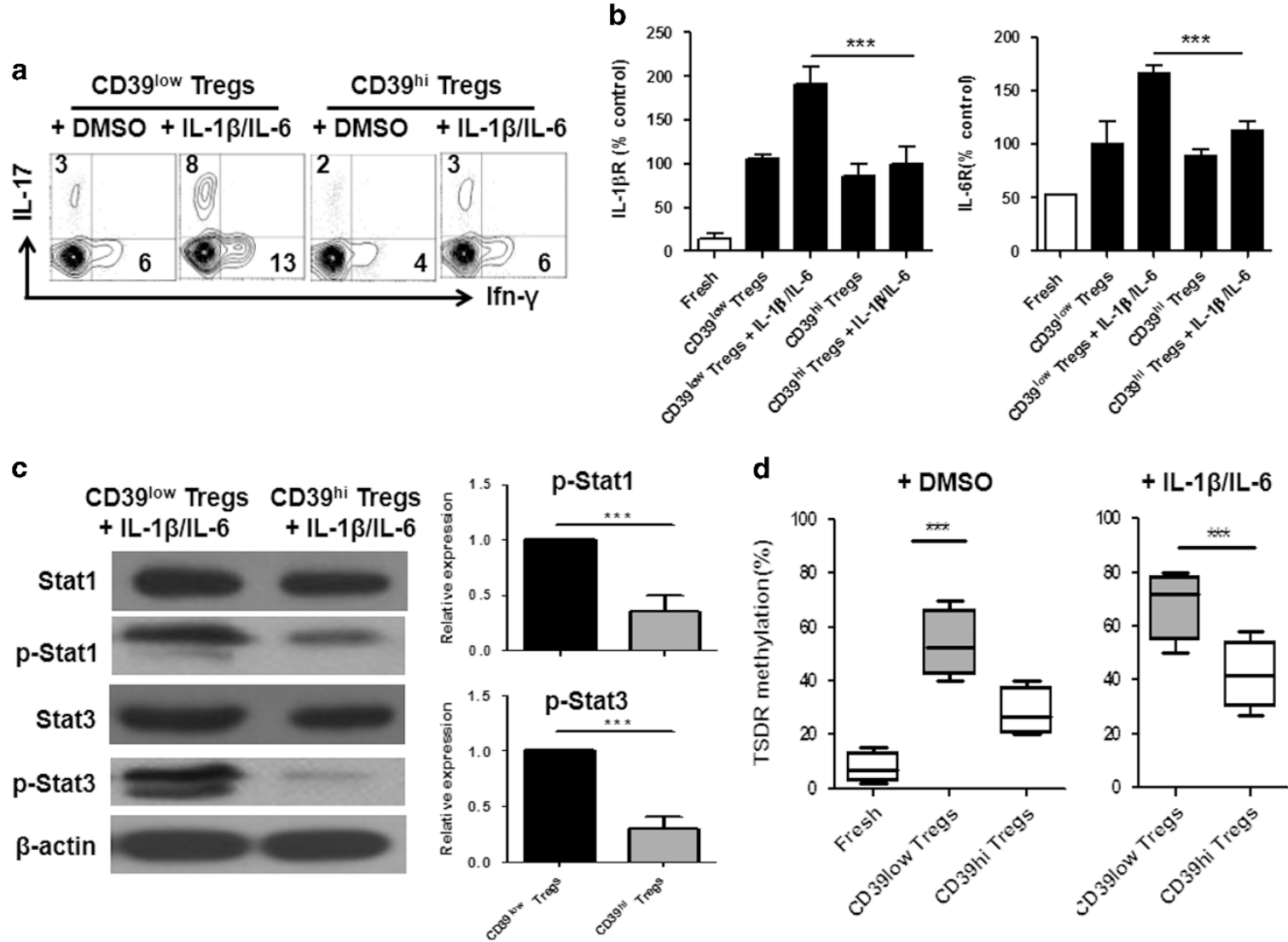

Figure 2 CD39hi Tregs decreased IL-1 $\beta$ R and IL-6R expression and downstream molecule activation and modulated CpG methylation when stimulated with IL-1 $\beta$ and IL-6 compared with CD39low Tregs. CD39hi Tregs and CD39low Tregs were stimulated with IL-1 $\beta$ and IL-6 for 3 days. Then, the expression of IL-1 $1 \beta \mathrm{R}$ and IL-6R and downstream signalling molecules was examined. (a) Representative IFN- $\gamma$ and IL-17 frequencies of Tregs in the presence of IL- $1 \beta$ and IL- 6 from four separate experiments. (b) The expression of IL- $1 \beta$ receptors and IL- 6 receptors determined by RT-PCR. Black bars represent day 3 post expansion with TCR stimulus and white bars represent ex vivo cells. The CD39low Treg vehicle was arbitrarily assigned as 1 and the other values were normalized to this value. (c) Left panel: the expanded Tregs were stimulated with IL-1 $\beta$ and IL- 6 for 30 min and the levels of phosphorylated STAT1 and STAT3 were determined by western blotting. The data are representative of four separate experiments with similar results. Right panel: relative phosphorylation level (mean \pm s.e.m. of four separate experiments) of STAT1 and STAT3 normalized to the expression of the total STAT1 and STAT3 protein. The DMSO vehicle was arbitrarily assigned as 1 and the other values were normalized to this value. (d) Methylation status of CpG motifs of the TSDR of the Foxp3 locus in the aforementioned harvested Tregs was detected with bisulfite sequencing PCR. The percentages are the average methylation values within the TSDR. The left panel shows the methylation level under cytokine-free conditions, whereas inflammatory cytokines are added in the right panel. The data are shown as the mean \pm s.e.m. of four independent experiments. ${ }^{* *} P<0.01,{ }^{* * *} P<0.001$.

the mechanism by which the CD39low Tregs were less stable than their CD39hi counterparts following IL-1 $\beta$ and IL-6 stimulation, we measured STAT1, p-STAT1, STAT3 and p-STAT3 protein expression in the CD39 $9^{\text {low }}$ and CD39hi Tregs subjected to IL-1 $\beta$ and IL-6 stimulation. The STAT1 and STAT3 phosphorylation levels were substantially lower in the CD39 ${ }^{\text {hi }}$ Tregs than in the CD39 ${ }^{\text {low }}$ Tregs (Figure 2c). The epigenetic regulation of the Foxp3 locus through the demethylation of $\mathrm{CpG}$ islands has been recognized as an important hallmark for Treg stability and functionality. ${ }^{12}$ We used bisulfite sequence analysis to examine the methylation status of the CD39 $9^{\text {low }}$ Tregs and CD39hi Tregs in the presence or absence of IL-1 $\beta$ and IL-6. As expected, freshly isolated Tregs exhibited few methylated CpG sites (94.8 $\pm 1.4 \%)$; after cell expansion and sorting, the CD39low Tregs showed a lower methylation level $(83.3 \pm 2.7 \%)$ than the CD39 $9^{\text {hi }}$ Tregs $(84.9 \pm 3.2 \%)$. When IL- $1 \beta$ and IL- 6 were added to the culture, the CD39 ${ }^{\text {low }}$ Tregs exhibited a significant decrease $(72.6 \pm 4.6 \%)$ in methylated CpG sites, whereas the methylation level of the CD39hi Tregs $(77.6 \pm 3.2 \%)$ was better maintained (Figure 2d).

\section{Human CD39hi Tregs but not CD39 ${ }^{\text {low }}$ Tregs protected against xeno-GvHD in vivo}

Our in vitro findings that CD39hi Tregs were more stable and functional than CD39low Tregs prompted us to investigate whether the CD39hi Tregs were also better than the CD39low Tregs in vivo. We analysed the immunosuppressive capacities of the CD39hi and CD39low Tregs in the mouse xeno-GvHD model with the Tregs serving as the major rescued population. Co-transfer of the CD39hi Tregs with PBMCs significantly prolonged mouse survival compared with the CD39low Treg and PBMC-alone groups, with a mean survival of $60.0 \pm 7.44$ days compared with $19.8 \pm 7.33$ and $23.8 \pm 7.33$ days, respectively (Figure 3a). Unexpectedly, the CD39low Tregs lost their protective ability, indicating that the CD39low Tregs were not stable and functional in vivo. 
Correspondingly, significantly lower expansion of allo-human $\mathrm{T}$ cells and delayed severe weight loss were observed after CD39hi Treg transfusion compared with the CD39 low Treg group (Figures $3 \mathrm{~b}$ and c). Regarding inflammatory cytokines, CD39 ${ }^{\text {hi }}$ Treg treatment led to a reduction in IL-6, IL-17, IFN- $\gamma$ and TNF$\alpha$ production by $\mathrm{CD}^{+}{ }^{+}$splenic $\mathrm{T}$ lymphocytes, whereas the cells produced a similar amount of IL-4 compared with the CD39low group (Supplementary Figure 2A). Self-tolerance is maintained by stable thymic Foxp $3^{+} \mathrm{CD} 4^{+} \mathrm{T}$ cells with a higher affinity for selfantigens. To test the stability of the injected Tregs, both CD39hi and CD39 ${ }^{\text {low }}$ Tregs were labelled with CFSE before injection into xenoGvHD model mice. After 7 days, the mice were euthanized and the cells isolated from the spleen were analysed using flow cytometry. Notably, we observed a marked decrease in Foxp3 expression in the CD39low Treg group compared with the CD39hi Treg group (Supplementary Figure 2B). In contrast, the CD39hi Tregs expressed less IL-17 than the CD39low Tregs (Figure 3d). To investigate the pathogenic role of the injected Tregs, $\mathrm{CFSE}^{+}$cells were sorted from the spleen and lymph nodes (LNs) 1 week after Treg injection. The RT-PCR analysis revealed that the CD39low Tregs expressed much higher IL-17 mRNA expression levels than the CD39hi group (Figure $3 \mathrm{e}$ ). We hypothesized that the self-reactive $\mathrm{T}$ cells derived from the $\mathrm{CD}^{+} \mathrm{Foxp}^{+} \mathrm{T}$ cells after the loss of Foxp3 expression significantly promoted the inflammatory effect. The CD39low Tregs proliferated more in vivo than the CD39hi Tregs, suggesting that a higher amount of converted $\mathrm{T}$ cells were detected 1 week after injection with the CD39 ${ }^{\text {low }}$ Tregs (Figure 3f).

The IL-2/IL-6 balance played an important role in stabilizing CD39 ${ }^{\text {low }}$ Treg functions in vivo

IL-2, which is a cytokine with pleiotropic effects, is critical for immune cell activation and peripheral tolerance. ${ }^{13}$ IL-2 limits $\mathrm{CD} 4^{+} \mathrm{CD} 8^{+} \mathrm{IL}-17^{+} \mathrm{T}$ cells and protects lupus-prone mice from multiple end-organ damage. ${ }^{14}$ In addition, iTregs promoted tolerance in acute-GvHD mice following rapamycin and IL-2 treatment in vivo. ${ }^{15}$ We analysed the immunosuppressive capacity of the administration of CD39 ${ }^{\text {low }}$ Treg in vivo with rhIL-2 $\left(5 \times 10^{4}\right.$ IU/mouse) every 3 days in xeno-GvHD mice. ${ }^{16}$ Co-transfer of the CD39low Tregs with rhIL-2 significantly prolonged mouse survival compared with the CD39low Treg group and the PBS treatment

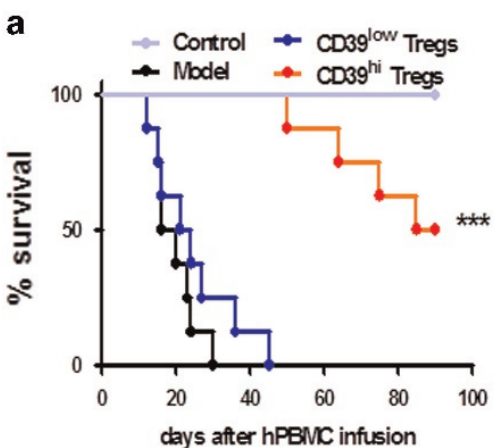

d

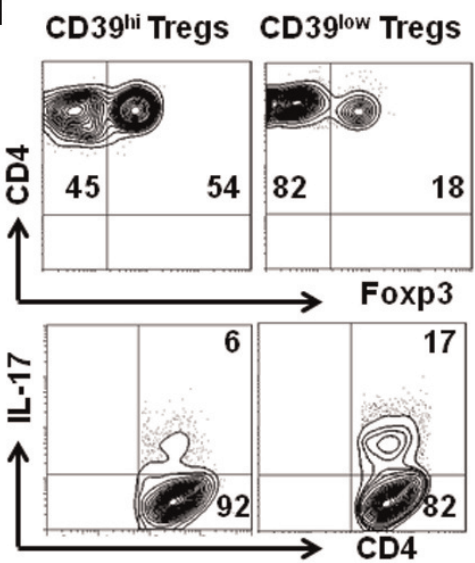

b

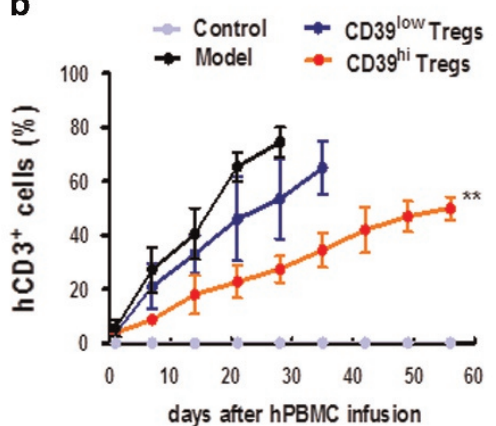

e

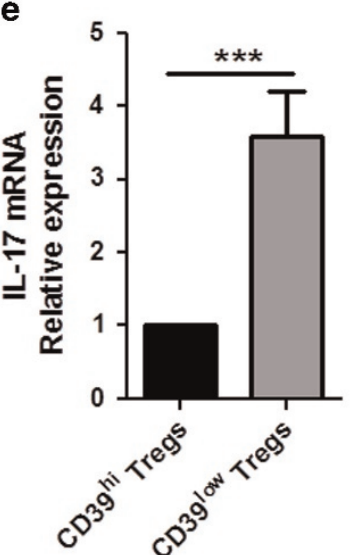

f
C
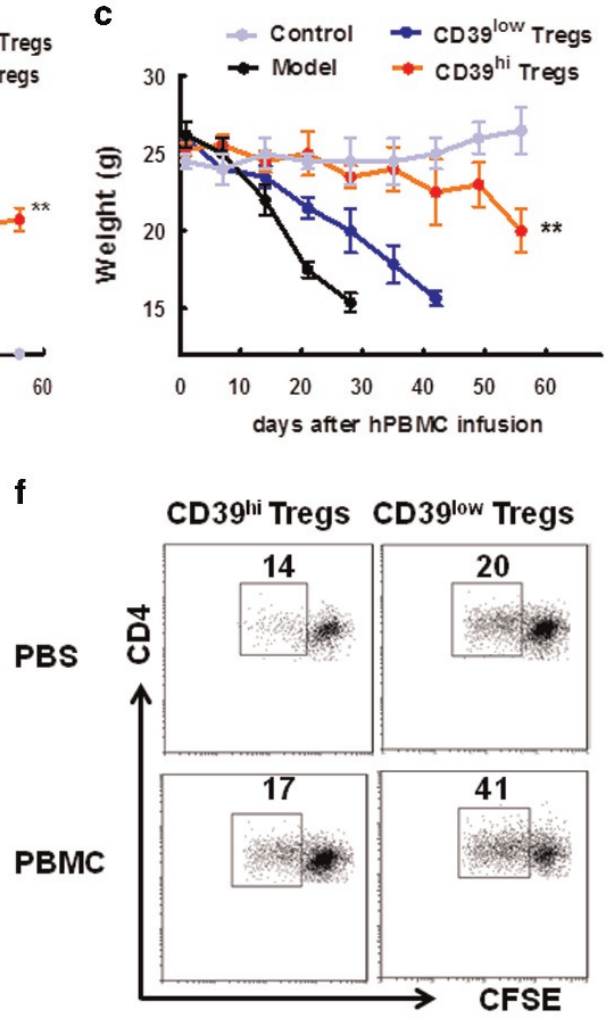

Figure 3 CD39hi Tregs increased the survival of xeno-GvHD mice and CD39low Tregs reverted into Th17 cells in vivo. A total of $20 \times 10^{6}$ freshly isolated human PBMCs were injected intravenously into NOD/SCID mice. Some mice were co-injected with $5 \times 10^{6}$ CD39hi Tregs or $5 \times 10^{6}$ CD39low Tregs with the PBMCs. The CD39hi Tregs or CD39low Tregs were obtained as described above. Mice that received only PBS injection were used as the control group. (a) The survival of the xeno-GvHD mice is shown. Kaplan-Meier survival curves depict the percentage of live mice. (b) Peripheral blood was collected weekly, stained with anti-human CD3 antibodies and quantified by flow cytometry. (c) The average weight is shown as the mean \pm s.e.m. (d) Representative contour plots of $\mathrm{CD} 4^{+} \mathrm{Foxp} 3^{+}$and $\mathrm{CD} 4^{+} \mathrm{IL}-17^{+}$cells out of the CFSE + cell population of spleen lymphocytes. (e) Relative IL-17 mRNA expression of CFSE ${ }^{+}$cells sorted from the spleens of CD39low and CD39hi treated xeno-GvHD group mice 1 week after xeno-GvHD. (f) In vivo proliferative responses of CD39hi Tregs or CD39low Tregs from the spleens of the xeno-GvHD mice. The numbers indicate the frequency of proliferating cells determined by the CFSE dilution. The weight loss data are presented as the mean \pm s.e.m. from three independent experiments ( $n=8$ for each group). ${ }^{*} P<0.05 ;{ }^{* *} P<0.01 ;{ }^{* *} P<0.001$. 
group, with a mean survival of $47.0 \pm 7.44$ days compared with $22.8 \pm 7.33$ days and $18 \pm 7.33$ days, respectively (Figure 4a). As expected, IL-2 controlled the weight loss of the xeno-GvHD mice in the CD39low Treg group (Figure 4b). To investigate the conversion of the CD39low Tregs, CFSE-labelled Tregs were injected into the xeno-GvHD mouse model. After 1 week, $\mathrm{CFSE}^{+}$ cells were sorted from the spleens and LNs, and IL-17 mRNA expression was evaluated via RT-PCR. IL-17 mRNA expression was much higher in the CD39low Treg group than the CD39low Treg and rhIL-2 groups (Figure 4c), indicating that IL-2 sustained the stability of the CD39 $9^{\text {low }}$ Tregs in the inflammatory environment. Treatment of the CD39low Tregs with rhIL-2 led to lower IL-6, IL-17 and IFN- $\gamma$ expression by $\mathrm{CD}^{+}{ }^{+}$splenic T lymphocytes, whereas no significant difference in IL-4 expression was detected between the groups (Figure $4 \mathrm{~d}$ ). Inflammatory factors and an imbalance of homeostasis contribute to the severity of autoimmune diseases. Interleukin-6 (IL-6), which is a cytokine that plays an important role in immune responses, has been implicated in the pathogenesis of autoimmune diseases. ${ }^{17}$ Therefore, we analysed the immunosuppressive capacity of administration of the CD39low Tregs in vivo with the humanized IL-6R inhibitor tocilizumab $(8 \mathrm{mg} / \mathrm{kg})$ in xeno-GvHD mice. Treatment with the IL-6R inhibitor alone increased the survival and reduced the severity of xeno-GvHD (Supplementary Figures $3 \mathrm{~A}$ and $\mathrm{B}$ ). Importantly, the expansion of human $\mathrm{CD}^{+} \mathrm{T}$ cells was significantly decreased (Supplementary Figure 3C). We also analysed the conversion of the injected Tregs as described in Figure 4c. The results revealed that the CD39low Tregs expressed higher IL-17 mRNA levels compared with the CD39low Treg and the tocilizumab treated groups (Supplementary Figure 3D). Treatment of the CD39 ${ }^{\text {low }}$ Tregs with tocilizumab also led to lower IFN- $\gamma$ expression by $\mathrm{CD}^{+}{ }^{+}$splenic $\mathrm{T}$ cells compared with the CD39low Tregs alone, whereas no significant difference was detected for IL-6 and IL-17 expression between the groups (Supplementary Figure 4E).

\section{CD39hi Tregs but not CD25 ${ }^{\text {hi }}$ Tregs exhibited sustained} Foxp3 levels and functional abilities after long-term Treg expansion in vitro

Human Tregs need to be repeatedly expanded ex vivo to gain sufficient cell numbers for clinical use. Therefore, we investigated whether CD39hi Tregs showed stronger Foxp3 expression and functional activity during the long-term expansion process.
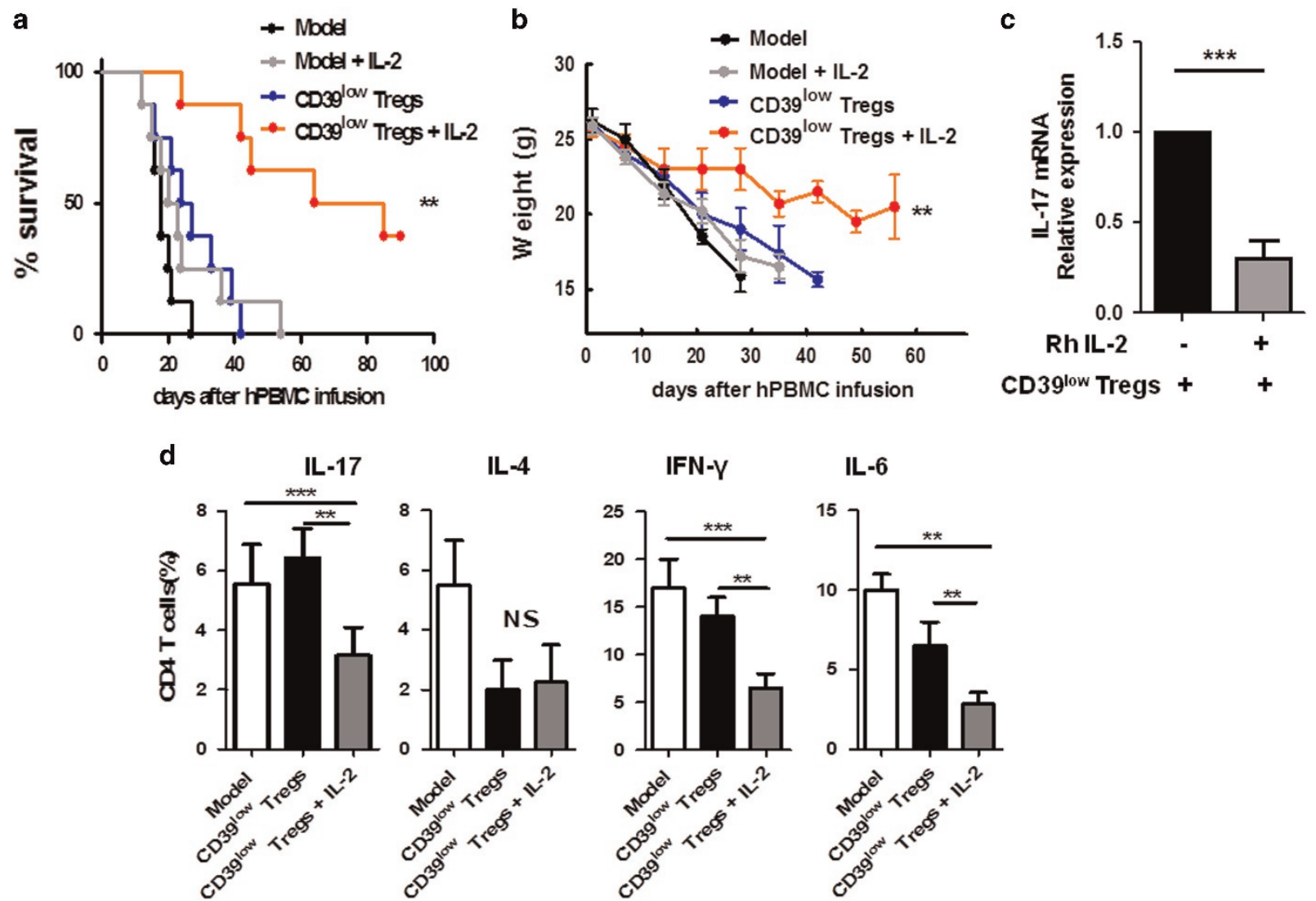

Figure 4 The IL-2/IL-6 balance played an important role in stabilizing CD39low Treg functions in vivo. A total of $20 \times 10^{6}$ freshly isolated human PBMCs were injected intravenously into NOD/SCID mice. Some mice were co-injected with $5 \times 10^{6}$ CD39low Tregs or $5 \times 10^{6}$ CD39low Tregs with recombinant IL-2. The CD39low Tregs were obtained as described above. (a) Survival and (b) average weights of the GvHD mice are shown following treatment with the CD39 low Tregs with the addition of recombinant IL-2. (c) The CD39low Tregs were stained with CFSE before recombinant IL-2 injection. After 1 week, CFSE ${ }^{+}$cells from the spleens and LNs of the xeno-GvHD mice were sorted. Relative IL-17 mRNA expression was detected by RT-PCR. (d) The mice were euthanized and cytokine expression in CD4+ splenic T cells was determined by flow cytometry staining, including IL-17, IL-4, IFN- $\gamma$ and IL- 6 . The data are shown as the mean \pm s.e.m. from four independent experiments ( $n=8$ for each group). ${ }^{* *} P<0.01 ; * * P<0.001$. 
Because CD25 ${ }^{\text {hi }}$ Tregs also showed a stronger protective effect compared with CD25 low Tregs in the treatment of collageninduced arthritis (CIA), ${ }^{18}$ we compared the effect of the CD25 ${ }^{\text {hi }}$ Tregs and CD39 ${ }^{\text {hi }}$ Tregs on Treg functions. CD $4^{+} \mathrm{CD} 25^{+} \mathrm{CD} 127^{-}$ $\mathrm{T}$ cells were sorted and cultured with anti-CD3/CD28 beads $(2: 1)$ for 12 days; then, the cells were re-stimulated with the beads for 9 days. IL-2 and rapamycin were added to the culture every 2 days (Supplementary Figure 4A). Rapamycin was added to facilitate long-term Treg culture and maintain Foxp3 expression. The cells were harvested and the CD25 $5^{\text {hi }}$ and CD25 low Tregs and CD39hi and CD39low Tregs were sorted (Supplementary Figure 4B). The CD39 ${ }^{\text {hi }}$ Tregs expressed higher Foxp3 levels than the CD39low Tregs $(88 \% \pm 4.2 \%$ vs $69 \% \pm 2.0 \%)$. Unexpectedly, the CD $25^{\mathrm{hi}}$ Tregs showed lower Foxp3 expression than the CD25 ${ }^{\text {low }}$ Tregs $(71 \% \pm 5.2 \%$ vs $89 \% \pm 3.1 \%)$. We also analysed CD39 expression in the $\mathrm{CD} 25^{\text {hi }}$ and $\mathrm{CD} 25^{\text {low }}$ Treg subsets. Our results revealed that the CD39 expression level in the CD25 $5^{\text {hi }}$ Tregs was slightly decreased compared with the CD25 low Tregs (Supplementary Figure 4B). Because the suppressive ability of Tregs is important for their function, CFSE co-culture assays were performed to estimate the functions of the four Treg subsets. The Tregs were co-incubated with CFSE-labelled fresh PBMCs in the presence of anti-CD3 beads at a low ratio (1:20). The CD39hi Tregs showed higher suppressive functions compared with the CD39low Tregs. In addition, we observed a stronger suppressive effect in the CD25 ${ }^{\text {low }}$ Tregs compared with the CD25 ${ }^{\text {hi }}$ Tregs (Supplementary Figures $4 \mathrm{C}$ and D).

Finally, we analysed the co-expression of CD25 and CD39 in the expanded Tregs. The results showed that most of the cells were CD25 and CD39 double positive after 21 days of culture, although the $\mathrm{CD} 4^{+} \mathrm{Foxp}^{+}$cells expressed higher CD39 levels than the $\mathrm{CD} 4^{+}$ Foxp $3^{-}$cells. There was no difference in CD25 expression between these Treg subsets (Supplementary Figure 5).

\section{DISCUSSION}

This study demonstrated that although CD39low Tregs lost their suppressive functions upon the addition of IL-1 $\beta$ and IL- 6 in vitro, the CD39hi Tregs sustained their suppressive ability and exhibited higher Foxp3 expression even within an inflammatory environment. We tested the functions of both Treg subsets in a xenoGvHD mouse model. The results revealed that mice injected with the CD39hi Tregs showed much slower weight loss and a higher survival index, thereby establishing the in vivo importance of $\mathrm{CD} 9^{+}$Tregs in regulatory $\mathrm{T}$ cell conversion. The western blotting and RT-PCR analysis demonstrated that the CD39 low Tregs exhibited much higher STAT1/STAT3 phosphorylation and CpG methylation levels than the CD39hi Tregs.

The balance between anti-inflammatory cytokines (IL-2) and pro-inflammatory cytokines (IL-1 $\beta$ and IL-6) regulates the differentiation of Treg and Th17 cells from naive T cells ${ }^{19,20}$ as well as Treg plasticity. ${ }^{21}$ Following the injection of rhIL-2 or the IL-6R inhibitor tocilizumab in vivo, the CD39low Tregs showed a much stronger protective ability and less pathogenic conversion than the Tregs from the groups that did not receive rhIL-2 or the IL-6R inhibitor injection. These data indicated that the IL-2 and IL-6 balance was highly related to the stability of the CD39 low Tregs in the xeno-GvHD model.
To date, studies have demonstrated that CD25 $5^{\text {hi }}$ Tregs are a more functional subset than their $\mathrm{CD} 25^{\text {low }}$ counterparts. CD25 $5^{\text {low }}$ Tregs lose their suppressive ability in the presence of inflammatory cytokines; ${ }^{18}$ however, unexpectedly, the CD25 ${ }^{\text {hi }}$ Tregs showed reduced Foxp3 expression and a reduced suppressive ability compared with the CD25 $5^{\text {low }}$ Tregs after 21 days of culture. Higher Foxp3 expression was observed in the CD39 ${ }^{\text {hi }}$ Tregs compared with the CD39low Tregs. The data from the in vitro suppressive assays were consistent with the aforementioned Foxp3 expression studies. Thus, CD25 may be an activation marker for T cells, especially stimulated Tregs. Stronger CD25 expression may be related to over-activation of the TCR, which will result in the loss of Foxp3 expression. Conversely, CD39 expression is closely related to regulatory $\mathrm{T}$ cells and higher CD39 expression may be an ideal marker for Treg stability and function. CD39 expression was increased in the CD25 ${ }^{\text {low }}$ Tregs but reduced in the CD25 ${ }^{\text {hi }}$ Tregs. Because Tregs are strictly dependent on IL-2, IL-2R expression may be modulated in the culture and may be dependent on the presence of IL-2. In addition, the adenosine produced by CD39hi Tregs may influence the level of CD25 expression. The data showed that CD39 sorting might be an effective procedure to improve the function of GMP-level longterm Treg production. Furthermore, these data imply that CD39 ${ }^{\text {low }}$ Tregs, which are more readily converted into inflammatory $\mathrm{T}$ cells in vitro and in vivo, should be depleted before clinical cell therapy to optimize the therapeutic benefits.

The results presented in this study suggest that the CD39/ adenosine pathway is important for the maintenance of the balance between the activation and regulation of immune responses. Studies have shown that CD39 is involved in many immune disorders, such as HIV. ${ }^{22,23}$ The $\mathrm{CD}^{2} 9^{+} \mathrm{CD} 4^{+}$Treg subsets demonstrated a strong suppressive ability in mouse EAE models. ${ }^{24}$ Human $\mathrm{CD} 4{ }^{+} \mathrm{CD} 39^{+}$Tregs produced adenosine upon co-expression with surface $\mathrm{CD} 73,{ }^{25,26}$ and adaptive Tregs were shown to suppress immune responses through adenosine. ${ }^{27}$ Different treatment regimens can increase CD39 expression in Tregs in vitro and in vivo. For example, a recent study showed that the percentage of functionally suppressive CD $39^{+}$Tregs increased as a result of anti-IL-6R treatment in both CIA and rheumatoid arthritis. ${ }^{28}$ A higher $\mathrm{CD} 39^{+} \mathrm{CD}^{2} 3^{+}$frequency was detected following treatment of tumours with TGF- $\beta,{ }^{29}$ and rapamycin promoted the demethylation of Tregs in the TSDR region and increased $\mathrm{CD}_{39}{ }^{+} \mathrm{Foxp}^{+}$expression and the suppressive activity. ${ }^{30,31}$ These findings at least partially explain why the protocols described above enhance Treg functions. Another report from our group showed that $\mathrm{CD}_{3}{ }^{+}$naive $\mathrm{T}$ cells were more readily skewed to the iTreg phenotype and possessed enhanced proliferative and suppressive abilities and resistance to cytokine expression. ${ }^{32}$ Although both protocols are efficient at generating functional and stable Tregs, we believe that our protocol reported here for sorting CD39hi Tregs after in vitro Treg expansion will be more applicable because the expanded Tregs will be subnormal for some patients; thus, sorting CD39hi Tregs will not only improve Treg functions but also provide a method to remedy the subnormality. Our findings in this study present a new understanding of Treg stability and a new method to improve Treg preparation before adoptive cell therapy. Further characterization of the mechanisms underlying the 
conversion and function of CD39 ${ }^{\text {low }}{ }^{2}$ oxp $3^{+} \mathrm{T}$ cells is needed for the development of therapeutic strategies for immune regulation.

\section{CONFLICT OF INTEREST}

The authors declare no conflict of interest.

\section{ACKNOWLEDGEMENTS}

This work was supported by the National Natural Science Fund 81509236 (XHW), National Natural Science Fund Outstanding Youth Fund 81522020 (LL), 863 Young Scientists Special Fund 81522020 (LL) and National Natural Science Fund 91442117 and 81571564 (LL) in China and Jiangsu Province's Outstanding Medical Academic Key Program RC2016 (LL).

1 Sakaguchi S, Sakaguchi N, Asano M, Itoh M, Toda M. Immunologic selftolerance maintained by activated $\mathrm{T}$ cells expressing $\mathrm{IL}-2$ receptor alphachains (CD25). Breakdown of a single mechanism of self-tolerance causes various autoimmune diseases. J Immunol 1995; 155: 1151-1164.

2 Li Z, Li D, Tsun A, Li B. FOXP3+ regulatory T cells and their functional regulation. Cell Mol Immunol 2015; 12: 558-565.

3 Kasagi S, Zhang P, Che L, Abbatiello B, Maruyama T, Nakatsukasa $\mathrm{H}$ et al. In vivo-generated antigen-specific regulatory $\mathrm{T}$ cells treat autoimmunity without compromising antibacterial immune response. Sci Trans/ Med 2014; 6: 241ra78.

4 Kong $\mathrm{N}$, Lan Q, Chen M, Zheng T, Su W, Wang J et al. Induced T regulatory cells suppress osteoclastogenesis and bone erosion in collagen-induced arthritis better than natural $\mathrm{T}$ regulatory cells. Ann Rheum Dis 2012; 2012: 6.

5 Ganguly S, Ross DB, Panoskaltsis-Mortari A, Kanakry CG, Blazar BR, Levy RB et al. Donor CD4+ Foxp3+ regulatory T cells are necessary for post-transplantation cyclophosphamide-mediated protection against GVHD in mice. Blood 2014; 124: 2131-2141.

6 Sawant DV, Vignali DA. Once a Treg, always a Treg? Immunol Rev 2014; 259: 173-191.

7 Della Latta V, Cabiati M, Rocchiccioli S, Del Ry S, Morales MA. The role of the adenosinergic system in lung fibrosis. Pharmacol Res 2013; 76: 82-89.

8 Deaglio S, Dwyer KM, Gao W, Friedman D, Usheva A, Erat A et al. Adenosine generation catalyzed by CD39 and CD73 expressed on regulatory T cells mediates immune suppression. J Exp Med 2007; 204: 1257-1265.

9 Lu L, Lan Q, Li Z, Zhou X, Gu J, Li Q et al. Critical role of all-trans retinoic acid in stabilizing human natural regulatory $T$ cells under inflammatory conditions. Proc Natl Acad Sci USA 2014; 111: E3432-E3440.

10 Zhou X, Kong N, Wang J, Fan H, Zou H, Horwitz D et al. Cutting edge: all-trans retinoic acid sustains the stability and function of natural regulatory T cells in an inflammatory milieu. J Immunol 2010; 185: 2675-2679.

11 Sawa S, Kamimura D, Jin GH, Morikawa H, Kamon H, Nishihara M et al. Autoimmune arthritis associated with mutated interleukin (IL)-6 receptor gp130 is driven by STAT3/IL-7-dependent homeostatic proliferation of CD4+ T cells. J Exp Med 2006; 203: 1459-1470.

12 Floess S, Freyer J, Siewert C, Baron U, Olek S, Polansky J et al. Epigenetic control of the foxp3 locus in regulatory T cells. PLoS Biol 2007; 5: e38.

13 Sauzullo I, Mastroianni CM, Mengoni F, Ermocida A, Mascia C, Salotti $\mathrm{A}$ et al. Long-term IFN-gamma and IL-2 response for detection of latent tuberculosis infection in healthcare workers with discordant immunologic results. J Immunol Methods 2014; 414: 51-57.

14 Mizui M, Koga T, Lieberman LA, Beltran J, Yoshida N, Johnson MC et al. IL-2 protects lupus-prone mice from multiple end-organ damage by limiting CD4-CD8- IL-17-producing T cells. J Immunol 2014; 193: 2168-2177.

15 Zhang P, Tey SK, Koyama M, Kuns RD, Olver SD, Lineburg KE et al. Induced regulatory $\mathrm{T}$ cells promote tolerance when stabilized by rapamycin and IL-2 in vivo. J Immunol 2013; 191: 5291-5303.
16 Zhang X, Wei HX, Rui S, Wei H, Tian Z. Opposite effects of high and low doses of interleukin-2 on T cell-mediated hepatitis in mice (interleukin-2 on hepatitis). Hepatol Int 2010; 4: 641-648.

17 Makrilakis K, Fragiadaki K, Smith J, Sfikakis PP, Kitas GD. Interrelated reduction of chemerin and plasminogen activator inhibitor-1 serum levels in rheumatoid arthritis after interleukin- 6 receptor blockade. Clin Rheumatol 2014: 34: 419-427.

18 Komatsu N, Okamoto K, Sawa S, Nakashima T, Oh-hora M, Kodama T et al. Pathogenic conversion of Foxp3+ T cells into TH17 cells in autoimmune arthritis. Nat Med 2014; 20: 62-68.

19 Yang XP, Ghoreschi K, Steward-Tharp SM, Rodriguez-Canales J, Zhu J, Grainger JR et al. Opposing regulation of the locus encoding IL-17 through direct, reciprocal actions of STAT3 and STAT5. Nat Immunol 2011; 12: 247-254.

20 Park BV, Pan F. The role of nuclear receptors in regulation of Th17/Treg biology and its implications for diseases. Cell Mol Immunol 2015; 12: 533-542.

21 Tang Q, Adams JY, Penaranda C, Melli K, Piaggio E, Sgouroudis E et al. Central role of defective interleukin-2 production in the triggering of islet autoimmune destruction. Immunity 2008; 28: 687-697.

22 Leal DB, Streher CA, Bertoncheli Cde M, Carli LF, Leal CA, da Silva JE et al. HIV infection is associated with increased NTPDase activity that correlates with CD39-positive lymphocytes. Biochim Biophys Acta 2005; 1746: 6 .

23 Nikolova M, Carriere M, Jenabian MA, Limou S, Younas M, Kök A et al. CD39/adenosine pathway is involved in AIDS progression. PLoS Pathog 2011; 7: e1002110.

24 Wang Y, Begum-Haque S, Telesford KM, Ochoa-Reparaz J, Christy M, Kasper EJ et al. A commensal bacterial product elicits and modulates migratory capacity of CD39 CD4 T regulatory subsets in the suppression of neuroinflammation. Gut Microbes 2014; 5: 552-561.

25 Schuler PJ, Saze Z, Hong CS, Muller L, Gillespie DG, Cheng D et al. Human $\mathrm{CD} 4(+) \mathrm{CD} 39(+)$ regulatory $T$ cells produce adenosine upon coexpression of surface CD73 or contact with CD73(+) exosomes or CD73 (+) cells. Clin Exp Immunol 2014; 177: 13.

26 Otsuka A, Hanakawa S, Miyachi Y, Kabashima K. CD39: a new surface marker of mouse regulatory gammadelta T cells. J Allergy Clin Immunol 2013; 132: 1448-1451.

27 Mandapathil M, Szczepanski MJ, Szajnik M, Ren J, Jackson EK, Johnson JT et al. Adenosine and prostaglandin E2 cooperate in the suppression of immune responses mediated by adaptive regulatory T cells. J Biol Chem 2010; 285: 27571-27580.

28 Thiolat A, Semerano L, Pers YM, Biton J, Lemeiter D, Portales P et al. Interleukin-6 receptor blockade enhances CD39+ regulatory $T$ cell development in rheumatoid arthritis and in experimental arthritis. Arthritis Rheumatol 2014; 66: 273-283.

29 Ryzhov SV, Pickup MW, Chytil A, Gorska AE, Zhang Q, Owens P et al. Role of TGF-beta signaling in generation of CD39+CD73+ myeloid cells in tumors. J Immunol 2014; 193: 3155-3164.

30 Lu Y, Wang J, Gu J, Lu H, Li X, Qian X et al. Rapamycin regulates iTreg function through CD39 and Runx1 pathways. J Immunol Res 2014; 2014: 989434.

31 Akimova T, Kamath BM, Goebel JW, Meyers KE, Rand EB, Hawkins A et al. Differing effects of rapamycin or calcineurin inhibitor on T-regulatory cells in pediatric liver and kidney transplant recipients. Am J Transplant 2012; 12: 3449-3461.

32 Lu Y, Wang X, Gu J, Lu H, Zhang F, Li X et al. iTreg induced from CD39 (+) naive $T$ cells demonstrate enhanced proliferate and suppressive ability. Int Immunopharmacol 2015; 28: 925-930.

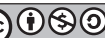

This work is licensed under a Creative Commons Attribution-NonCommercial-ShareAlike

4.0

International License. The images or other third party material in this article are included in the article's Creative Commons license, unless indicated otherwise in the credit line; if the material is not included under the Creative Commons license, users will need to obtain permission from the license holder to reproduce the material. To view a copy of this license, visit http:// creativecommons.org/licenses/by-nc-sa/4.0/

Supplementary Information for this article can be found on the Cellular \& Molecular Immunology website (http://www.nature.com/cmi) 